\title{
Robotic abdominal wall repair: adoption and early outcomes in a large academic medical center
}

\author{
Xavier Pereira ${ }^{1}$ - Diego L. Lima ${ }^{5}$. Patricia Friedmann ${ }^{2,3,4}$. Gustavo Romero-Velez ${ }^{1} \cdot$ Cosman C. Mandujano $^{1}$. \\ Vicente Ramos-Santillan ${ }^{1} \cdot$ Ana Garcia-Cabrera ${ }^{1} \cdot$ Flavio Malcher $^{5}$
}

Received: 14 October 2020 / Accepted: 8 May 2021 / Published online: 20 May 2021

(C) The Author(s), under exclusive licence to Springer-Verlag London Ltd., part of Springer Nature 2021

\begin{abstract}
Robotic-assisted abdominal wall repair (RAWR) has seen an exponential adoption over the last 5 years. Skepticism surrounding the safety, efficacy, and cost continues to limit a more widespread adoption of the platform. We describe our initial experience of 312 patients undergoing RAWR at a large academic center. A retrospective review of all patients undergoing any RAWR from July 1, 2016 to March 18, 2020 was completed. Patient specific, operation specific, and 30-day outcomes specific data were collected. Univariate analysis and multivariate logistic regression were used to assess factors associated with 30-day complications. There was a steady adoption of RAWR over the study period. A total of 312 patient were included, 138 (44\%) were abdominal wall repairs and $174(56 \%)$ were inguinal repairs. The mean age of the cohort was 54.2 years (SD 16), 69\% were males, and the mean BMI was $29 \mathrm{~kg} / \mathrm{m}^{2}$ (SD 4.8). There were two reported intraoperative events and nine operative conversions. 60 patients had at least one complication at 30-days. These include: 52 seromas, 4 hematomas, 2 surgical-site infections, 1 deep venous thrombus, and 1 recurrence at 30-days. BMI, type of hernia, and sex were not associated with complications at 30-days. The use of absorbable mesh, longer hospital stay, operative conversion, previous repair, and expert hernia surgeon were significant predictors of 30-day complications. Age, operative conversion, and previous repair were the only predictors of 30-day complications on multivariate regression. Our initial experience of 312 patients demonstrates the adoption and comparable short-term outcomes for a wide variety of robotic-assisted hernia repairs.
\end{abstract}

Keywords Robotic surgery $\cdot$ Hernia $\cdot$ Minimally invasive $\cdot$ Inguinal hernia $\cdot$ Ventral hernia $\cdot$ Safety $\cdot$ Adoption

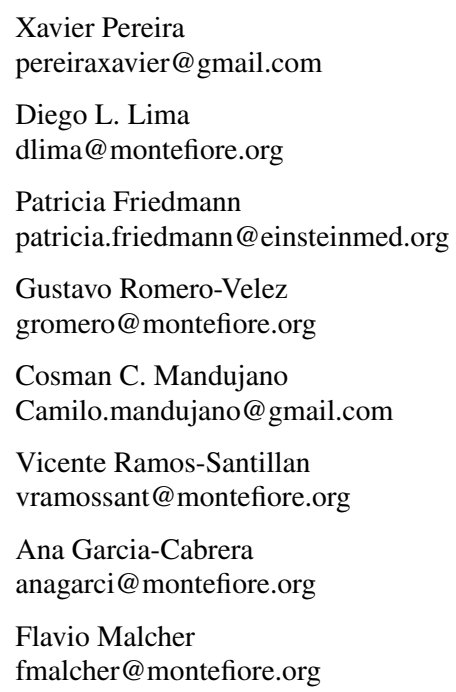

1 Department of General Surgery, Montefiore Medical Center and the Albert Einstein College of Medicine, 182 E. 210th Street (Lower Level), Bronx, NY 10467, USA

2 Department of General Surgery, Albert Einstein College of Medicine, 1300 Morris Park Avenue, Block Building Room 613, Bronx, NY 10461, USA

3 Departments of Cardiothoracic, Albert Einstein College of Medicine, 1300 Morris Park Avenue, Block Building Room 613, Bronx, NY 10461, USA

4 Department of Vascular Surgery, Albert Einstein College of Medicine, 1300 Morris Park Avenue, Block Building Room 613, Bronx, NY 10461, USA

5 Department of General Surgery, Montefiore Medical Center and the Albert Einstein College of Medicine, 1825 Eastchester Rd, 2N Floor General Surgery Office, Bronx, NY 10461, USA 


\section{Background}

Robotic-assisted abdominal wall repair (RAWR) is growing at an exponential rate [1]. In fact, no other type of surgery has seen a faster adoption of the robotic platform than hernia repair over the last decade [1]. However, a wider adoption of robotic hernia repair continues to meet skepticism as its safety, efficacy, cost, and ability to provide equal outcomes continue to be challenged. This skepticism stems from multiple factors [2]. For one, the availability of durable, safe, and relatively cheap open hernia repairs such as the Lichtenstein tension free repair for inguinal hernias or the multiple accepted component separation techniques for even the most complex abdominal wall (AW) hernias. Furthermore, the paucity of data on robotic hernia repair has prompted some to draw comparisons between laparoscopic and robotic hernia surgery. This may be problematic because the principles of these two surgical modalities have some important differences and the outcomes may not directly translate [3, 4].

Minimally invasive hernia repair touts shorter hospital stays, faster return to work, less post-operative pain, and a decreased incidence of surgical-site infections (SSI) [5]. The robotic platform offers some key advantages over laparoscopy. For the surgeon, a three-dimensional view of the operating field and articulating instruments allow for a more precise and ergonomic operating experience. These features allow for improved intra-corporeal fascial closure, extraperitoneal placement of mesh, more precise myofascial release, and more natural intraperitoneal suturing [6]. It is evident that robotic hernia repair represents the natural progression of minimally invasive hernia surgery [7].

Even with all of the technical advances that robotics offers over laparoscopy, many questions regarding outcomes, cost-benefit, and safety are yet to be answered [8, 9]. Nonetheless, abdominal wall experts have continued to advocate for the use of the robotics platform citing its many advantage, which is driving an interest in ongoing comparative outcomes research [10-12]. With this study, we aim to describe our initial experience of 312 patients undergoing robotic hernia repair at a large, multi-center academic hospital. In addition, we describe the experience in establishing an abdominal wall program dedicated to complex hernia repair and its impact on robotic hernia repair. Our focus on the safety, rate of adoption, and early outcomes may be particularly relevant as more institutions begin to embrace robotic hernia repair. We hypothesize that the adoption of the robotic platform for hernia repair represents a consistent progression of minimally invasive hernia repair with comparable short-term outcomes.

\section{Methods}

We conducted a retrospective review of all patients undergoing RAWR at a single academic institution from July 1, 2016 to March 18, 2020. Given our geographic location within the Bronx, NY we serve one of the most diverse regions in the United States [13]. Our patient population is rather unique and faces a high prevalence of poverty, smoking, diabetes, obesity, and a number of other comorbid conditions. Forty-two percent of patients in the Bronx are on Medicaid, $7 \%$ are on Medicare, and $8 \%$ of the population remain uninsured [14].

A comprehensive search of all hernia surgeries at our tertiary medical center was conducted and cases were classified by surgical modality as either open, laparoscopic, or robotic hernia repair. This study focuses specifically on the robotic hernia cohort. As such, a comprehensive chart review was conducted using our hospital's electronic medical records (EMR). Patients aged $>18$ years of age undergoing either inguinal or abdominal wall hernia repair were included. Six patients who underwent combined inguinal and abdominal wall repairs were excluded from this study. These hybrid cases could not be adequately categorized for statistical analysis without confounding the existing categorical groups. Patient characteristics were obtained from each patient's individual record. Operative details were obtained from the surgeon's operative note and the surgery specific encounter. Outcomes information was abstracted from subsequent encounters that included post-operative visit notes, emergency department (ED) notes, primary care physician notes, or post-operative imaging.

Our data collection was divided into three domains: patient specific, operation specific, and outcomes specific. Within our first domain, we collected patient characteristics including age, gender, body mass index (BMI), American Society of Anesthesiologists (ASA) class, all medical comorbidities, and history of previous hernia repair for the presenting hernia. A medical comorbidity was considered as a medical condition used in Charlson Comorbidity Index to calculate 10-year survival [15].

There is no consensus as to what constitutes an expert hernia surgeon. Following the guidelines of the European Hernia Society's (EHS) ACCESS project, an expert hernia surgeon can be defined as a surgeon who: is beyond the learning curve for all types of hernia surgery, including minimally invasive laparoscopic or robotic options in ventral and inguinal hernia; performs a proportionally larger number of hernia cases compared to other general surgeons, including complex abdominal wall repairs; participates in the education of other surgeons on the topic of hernia repair; participates in national hernia database data collection with outcomes reported; is a 
part of an established referral center; is an active member of a national surgical society with a focus on abdominal wall; participates hernia related publications, book chapters, presentations, or lectures in indexed journals and/or regional and national meetings per year, and participates in monthly hernia morbidity, quality control, and multidisciplinary conferences $[16,17]$. At our institution, only one surgeon met the criteria outlined above.

For operative information, we collected the length of each operation, lead surgeon, number and types of hernias repaired, presence and type of intraoperative complication, size of primary and subsequent defects for non-inguinal hernias, operating room (OR) conversion to open or laparoscopic modalities, operative technique, mesh brand, type, size, and fixation. Small bowel serosal tears were not considered an intraoperative complication as these do not necessarily alter the course of the planned operation. The length of the operation was quantified as the number of minutes between initial incision and the initial time marked as "dressing on" as noted by the anesthesia records or the circulating nurse records.

For post-operative information we collected data on hospital length of stay, type and rate of complication within 30 days, presentation to the ED or re-admission within 30-days, and the rate of follow-up. Complications were classified using the Clavien-Dindo (C-D) Classification System for post-operative events as follows: Grade I is any deviation from the expected post-operative course; Grade II is any deviation that requires medical treatment; Grade III is an deviation that requires procedural intervention, Grade IV is a life-threatening complication such as a single or multi-organ failure which requires treatment in the Intensive Care Unit; Grade V is a post-operative mortality [18]. Minor post-operative events were those that defined as Grades I-II of the C-D classification, while major post-operative events were those defined by Grades IV-V.

Data were summarized using counts and percentages for categorical variables and either means and standard deviations or median and range for continuous variables. Univariate associations with 30-day complications were assessed using Chi-squared and Fisher's exact tests for categorical variables and $t$ tests for continuous variables. Multivariate predictors of 30-day complications were estimated using multivariable logistic regression. $p$ values less than 0.05 were considered statistically significant. Data were analyzed using the Statistical Analysis System (SAS v9.4). This study was approved by the Institution Review Board (IRB \# 2020-11160).

\section{Results}

\section{Study period and case volume}

A total of 5266 abdominal wall repairs were completed over the 44-month study period at our institution. In the first quarter, 382 abdominal wall repairs were completed; 268 (71\%) were open, $102(27 \%)$ were laparoscopic, and $12(3 \%)$ were robotic assisted. By comparison, 271 abdominal wall cases were completed in the last quarter of the study period. Of these, 138 (51\%) were open, 32\% were laparoscopic, and $47(17 \%)$ were robotic assisted. The last quarter of our study, which ended in March of 2020, was affected the beginning of the COVID-19 pandemic. Nonetheless, similar changes in the distribution of cases were seen when comparing the first and last three quarters of the study period. Overall, despite there being a decrease in the total volume of cases, there was a steady adoption of RAWR over the 44-month study period (Fig. 1).

In July of 2018, 24 months after the start of our study period, a specialized multidisciplinary abdominal wall program was established at our institution. Prior to this time, all robotic cases were performed by only two surgeons. By the end of the study period, a total of seven surgeons were performing robotic hernia repairs, four of which were still in the learning phase $(<30$ cases completed) [19]. The participating surgeons had diverse backgrounds and experience level: one was an expert in abdominal wall reconstruction and hernia repair, two were trained in minimally invasive and bariatric surgery, three were non-fellowship trained general surgeons, and one was a transplant surgeon. The median number of years in practice was 12 .

\section{Patient cohort}

A total of 312 patients undergoing robotic hernia repair were identified and included in this study. The mean age among the cohort was 54 years (SD 16). $69 \%$ of patients were male, $31 \%$ were female. The mean BMI was $29 \mathrm{~kg} / \mathrm{m}^{2}$ (SD 4.8). As expected, inguinal hernia cases were predominantly male patients (56\%), while there was a larger proportion of female patients who underwent ventral hernia repairs (59\%). Most patients (59\%) had no comorbid conditions, $21 \%$ had at least one medical comorbidity, and $20 \%$ had two or more. Diabetes mellitus was the most prevalent comorbidity, present in $20 \%$ of patients. The majority of patients were ASA class II (58\%) and III (29\%). There were 50 (16\%) patients who presented with a recurrent hernia, more frequently seen in the ventral hernia $(21 \%)$ cohort as compared to the inguinal hernia group (12\%) (Table 1).

\section{Operative characteristics}

Operative characteristics are described in Table 2. Of the 312 RAWRs, 174 (56\%) were inguinal hernia repairs and $138(44 \%)$ were abdominal wall hernia repairs. Forty-two (28\%) of the inguinal hernias were bilateral. All inguinal hernia repairs were done via the trans-abdominal preperitoneal (TAPP) approach. Nearly all inguinal hernias (172) 


\section{HERNIA VOLUME - MONTEFIORE}

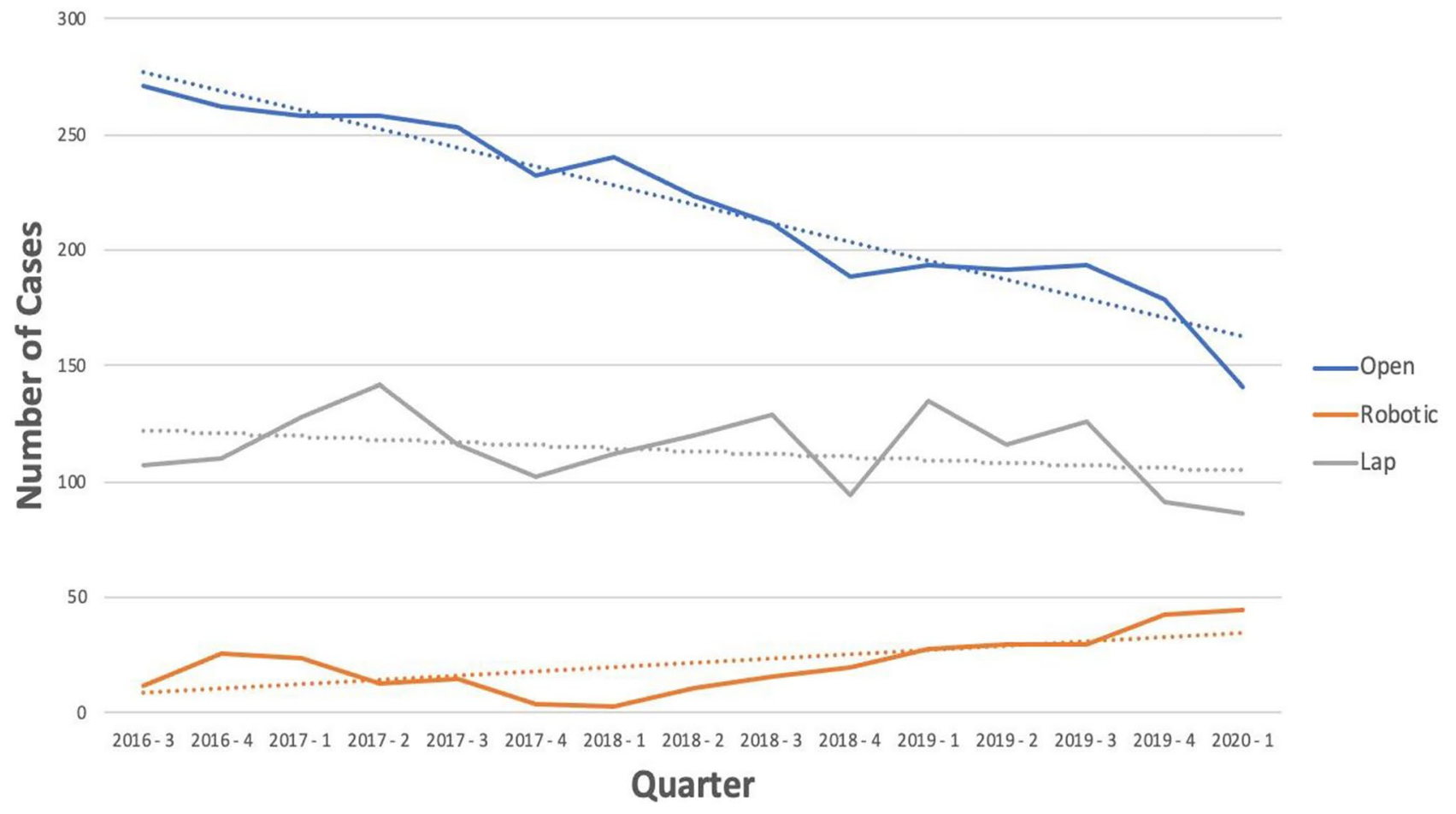

Fig. 1 Total hernia volume by modality at Montefiore Medical Center (MMC) between July of 2016 and March of 2020

were repaired using synthetic permanent mesh while only two were repaired using synthetic absorbable mesh. As expected, ventral hernias were approached with a variety of surgical techniques. By far, mesh placement in the extraperitoneal location was the most common with $26 \%$ of our entire cohort using this type of repair. The remainder of the ventral hernias were repaired with a variety of mesh location (preperitoneal, retro-muscular, or onlay) with or without component separation or transversus abdominis release (TAR) as outlined in Table 2. A total of 57 repairs were complete with the IPOM technique, 53 were completed with permanent coated mesh, 3 with synthetic absorbable mesh, and 1 biologic mesh. In the extraperitoneal position, one patient not have a mesh implanted due as intraoperative findings showed a well incorporated Marlex ${ }^{\circledR}$ mesh and no hernia. Table 3 includes a comprehensive list of all mesh used by location.

The mean primary defect size for abdominal wall hernias was $5.4 \mathrm{~cm}(1.0-30 \mathrm{~cm}, \mathrm{SD} 5.1)$. No defect size was reported for inguinal hernias. All hernia repairs included mesh reinforcement. At our institution, the IPOM plus, which is the placement of intraperitoneal mesh after closure of the hernia orifice, is technique is routinely used. There were two (4.5\%) reported intraoperative complications, one of which required a deviation from the planned surgery. Small bowel serosal tears were reported in 14 cases. None of these required a change in the planned operative technique. All 312 robotic cases in our study were completed using the da Vinci Si or $\mathrm{Xi}$ robotic systems (Intuitive Surgical, Inc. Sunnyvale, CA).

\section{Operative time and OR conversions}

Operative time followed an expected pattern with the longest cases coming from extraperitoneal ventral hernia repairs with a median operative time of $147 \mathrm{~min}$ (IQR 101 to 199). Unilateral inguinal hernias had the shortest operative times with a median of at $126 \mathrm{~min}$ (IQR 91-161). Shorter ventral hernia cases were related to intraperitoneal onlay mesh (IPOM) or preperitoneal mesh placement techniques, while longer cases had a higher frequency of component separation or retrorectus mesh placement. A total of nine (2.9\%) cases were converted to either open or laparoscopy. The low number of OR conversions limited our ability to examine factors leading to this intraoperative occurrence. Nonetheless, there were no identifiable factors like sex, BMI, ASA, type of surgeon, type of hernia repair, type of mesh, sex, or number of comorbidities related to intraoperative conversions (Table 4).

\section{Outcomes}

There was one major intraoperative event requiring a deviation of the planned surgery and prolonged admission. 
Table 1 Patient related information

\begin{tabular}{|c|c|}
\hline Total patients, $n(\%)$ & 312 \\
\hline Male & 214 \\
\hline Inguinal & 158 \\
\hline Abdominal wall & 56 \\
\hline Female & 98 \\
\hline Inguinal & 16 \\
\hline Abdominal wall & 82 \\
\hline Age (years); mean (SD) & $54(16)$ \\
\hline \multicolumn{2}{|l|}{ ASA class, $n(\%)$} \\
\hline I & $41(13)$ \\
\hline II & $182(58)$ \\
\hline III & $89(29)$ \\
\hline \multicolumn{2}{|l|}{ Comorbidities, $n(\%)$} \\
\hline Diabetes mellitus & $62(20)$ \\
\hline COPD & $7(2)$ \\
\hline MI & $8(3)$ \\
\hline $\mathrm{CHF}$ & $4(1)$ \\
\hline Liver disease & $15(5)$ \\
\hline HIV/AIDS & $7(2)$ \\
\hline Cancer & $33(11)$ \\
\hline \multicolumn{2}{|l|}{ No. of comorbidities, $n(\%)$} \\
\hline 0 & $183(59)$ \\
\hline 1 & $66(21)$ \\
\hline$\geq 2$ & $63(20)$ \\
\hline BMI $\left(\mathrm{kg} / \mathrm{m}^{2}\right)$; mean $(\mathrm{SD})$ & $29(4.8)$ \\
\hline Recurrent hernia, $n(\%)$ & $50(16)$ \\
\hline Inguinal & $21(12)$ \\
\hline Ventral & $29(21)$ \\
\hline \multicolumn{2}{|l|}{ Type of previous repair, $n$} \\
\hline Unknown & 32 \\
\hline Open & 5 \\
\hline Open with mesh & 6 \\
\hline Laparoscopic primary & 1 \\
\hline Laparoscopic with mesh & 4 \\
\hline Multiple previous repairs & 2 \\
\hline
\end{tabular}

ASA American Society Anesthesiology Classification, BMI body mass index

A second patient suffered a stroke on post-operative day 1 and required supportive care in the intensive care unit (ICU) but was eventually discharged home. Within the first 30 days, 34 (10.9\%) patients presented to the ED with a variety of complaints. Of these the 34 patients, 15 had wound related concerns like bruising, swelling, discharge, hematoma, or infection. The remaining patients presented to the ED with uncontrolled post-operative pain (10), urinary retention or hematuria (3), recurrence (1), constipation (1), and other unrelated causes like calf pain (1), stroke symptoms (1), and gastroenteritis (1). Of all the patients who presented to the ED, only 6 required
Table 2 Operative characteristics

\begin{tabular}{|c|c|}
\hline Total patients & 312 \\
\hline Inguinal & 174 \\
\hline Unilateral (4) & 126 \\
\hline Bilateral & 48 \\
\hline Abdominal wall & 138 \\
\hline Primary ventral & 85 \\
\hline Incisional & 52 \\
\hline Parastomal & 1 \\
\hline Operative time (min); mean (SD) & $139(67)$ \\
\hline Inguinal unilateral & $107(37)$ \\
\hline Inguinal bilateral & $159(63)$ \\
\hline Abdominal wall & $163(77)$ \\
\hline Intraoperative events, n (\%) & $2(1)$ \\
\hline Veress needle injury & 1 \\
\hline Thermal injury & 1 \\
\hline \multicolumn{2}{|l|}{ Operating surgeon, n (\%) } \\
\hline Hernia specialist & $110(35)$ \\
\hline Non-hernia specialist & $202(65)$ \\
\hline Operative conversion, $\mathbf{n}(\%)$ & $9(3)$ \\
\hline Laparoscopic & 4 \\
\hline Open & 5 \\
\hline Operative technique, n (\%) & $312(100)$ \\
\hline Inguinal & $174(56)$ \\
\hline TAPP & $174(56)$ \\
\hline Abdominal wall & $138(44)$ \\
\hline IPOM & $57(18)$ \\
\hline Extraperitoneal & $81(26)$ \\
\hline Retro-muscular & $45(14)$ \\
\hline Preperitoneal & $34(11)$ \\
\hline Inlay & $1(0.3)$ \\
\hline Unilateral component separation & 4 \\
\hline Bilateral component separation & 1 \\
\hline Transversus abdominis release (TAR) & 9 \\
\hline
\end{tabular}

readmissions; 2 for pain control, 2 for wound infection, and 2 for unrelated causes (gastroenteritis and stroke).

The cohort had a 30-day in-office follow-up rate of $89 \%$. Overall, there were 60 post-operative events, which includes those diagnosed in the ED as well as in the office. There was 1 early recurrence, 52 seromas, 2 SSI's, 4 hematomas, and 1 deep venous thrombus (DVT). (Table 4) There were 55 minor post-operative events (C-D Grade I-II) and 5 major post-operative (C-D Grade III-V) events. There were no deaths within 30 days (C-D Grade V). The overall 30-day complication rate was $19 \%$. Seromas were by far the most common post-operative complication with $52(17 \%)$ patients diagnosed in the postoperative period. These were all treated with supportive care and observation without additional morbidity (see Table 5). 
Table 3 Mesh type by location

\begin{tabular}{|c|c|}
\hline Extraperitoneal & $N$ \\
\hline Bard $^{\mathrm{TM}}$ Soft Mesh & 29 \\
\hline Covidien Progrip ${ }^{\mathrm{TM}}$ & 23 \\
\hline Prolene $\AA$ & 12 \\
\hline Covidien Symbotex ${ }^{\mathrm{TM}}$ & 10 \\
\hline BD Ventralight ${ }^{\mathrm{TM}} \mathrm{ST}$ & 3 \\
\hline BD Phasix ${ }^{\mathrm{TM}}$ Mesh & 4 \\
\hline BD Ventralex ${ }^{\mathrm{TM}}$ & 2 \\
\hline Marlex (in situ) & 1 \\
\hline GORE® SYNECOR & 1 \\
\hline ULTRAPRO ADVANCED ${ }^{\mathrm{TM}}$ & 1 \\
\hline \multicolumn{2}{|l|}{ IPOM } \\
\hline Covidien Symbotex ${ }^{\mathrm{TM}}$ & 46 \\
\hline BD Phasix ${ }^{\mathrm{TM}}$ Mesh & 3 \\
\hline Prolene® & 2 \\
\hline Parietene $^{\mathrm{TM}} \mathrm{DS}$ & 2 \\
\hline OviTex® & 1 \\
\hline BD Ventralight ${ }^{\mathrm{TM}}$ Echo & 1 \\
\hline BD Ventralight ${ }^{\mathrm{TM}}$ Comp & 1 \\
\hline Parietex ${ }^{\mathrm{TM}}$ Composite & 1 \\
\hline \multicolumn{2}{|l|}{ Inguinal } \\
\hline BD 3DMax ${ }^{\mathrm{TM}}$ Mesh & 99 \\
\hline Covidien Progrip ${ }^{\mathrm{TM}}$ & 69 \\
\hline GORE® SYNECOR & 3 \\
\hline BD Phasix ${ }^{\mathrm{TM}}$ Mesh & 2 \\
\hline Aborted/No mesh & 1 \\
\hline
\end{tabular}

On univariate analysis, there was no statistically significant relationship between complications at 30 days and the type of hernia (20 ventral, 21 inguinal), number of comorbid conditions (18 had 0 comorbidities, 27 had 1 comorbidity, and 19 had two or more comorbidities), or BMI (20 were $\mathrm{BMI}<30,16$ were $\mathrm{BMI} 30-35$, and 32 were $\mathrm{BMI}>35$ ). As expected, those patients who originally presented with recurrent hernias had a higher rate of complications at 30 days (17 had no previous repair, 37 had a previous repair). Intraoperatively, the use of absorbable or biologic mesh and OR conversion to laparoscopy or open modalities tended to have to a higher frequency of 30-day complications. Looking at BMI specifically, an increasing BMI did not have a correlation with OR conversions or 30-day complications in our cohort, even when controlling by type of hernia (Table 4).

A multivariate logistic regression analysis was conducted to determine factors associated with 30-day complications. The following variables were included in the regression model; hernia type (ventral vs. inguinal), ASA class, number of comorbidities, BMI, previous hernia repair, age, OR conversion to laparoscopy or open modalities, and type of surgeon (abdominal wall and hernia specialist versus others). Of these, only previous repair (aOR $2.38,95 \%$ CI
Table 4 Univariate analysis

\begin{tabular}{|c|c|c|c|c|c|}
\hline & \multicolumn{2}{|c|}{$\begin{array}{l}\text { OR conversion } \\
\text { Frequency }(\%)\end{array}$} & \multicolumn{3}{|c|}{$\begin{array}{l}\text { 30-day complication } \\
\text { Frequency }(\%)\end{array}$} \\
\hline & No & Yes & No & Yes & $p$ value \\
\hline$N=312$ & 303 & 9 & 252 & 60 & \\
\hline \multicolumn{6}{|l|}{ Type of hernia } \\
\hline Ventral & 96 & 4 & 80 & 20 & \multirow[t]{2}{*}{0.757} \\
\hline Inguinal & 98 & 2 & 79 & 21 & \\
\hline \multicolumn{6}{|l|}{ Sex } \\
\hline Female & 95 & 5 & 78 & 22 & \multirow[t]{2}{*}{0.649} \\
\hline Male & 98 & 2 & 80 & 20 & \\
\hline \multicolumn{6}{|l|}{ No. of comorbidities } \\
\hline 0 & 98 & 2 & 81 & 18 & \multirow[t]{3}{*}{0.286} \\
\hline 1 & 95 & 5 & 73 & 27 & \\
\hline$\geq 2$ & 95 & 5 & 81 & 19 & \\
\hline \multicolumn{6}{|l|}{ BMI } \\
\hline$<30$ & 97 & 3 & 80 & 20 & \multirow[t]{3}{*}{0.123} \\
\hline $30-35$ & 96 & 4 & 844 & 16 & \\
\hline$>35$ & 97 & 3 & 68 & 32 & \\
\hline \multicolumn{6}{|l|}{ ASA class } \\
\hline 1 & 98 & 2 & 90 & 10 & \multirow[t]{3}{*}{0.152} \\
\hline 2 & 96 & 4 & 80 & 20 & \\
\hline 3 & 99 & 1 & 75 & 25 & \\
\hline \multicolumn{6}{|l|}{ OR conversions } \\
\hline No conversion & & & 81 & 19 & \multirow[t]{3}{*}{0.004} \\
\hline Convert to lap & - & - & 75 & 25 & \\
\hline Convert to open & & & 20 & 80 & \\
\hline \multicolumn{6}{|l|}{ Surgeon type } \\
\hline Hernia specialist & 98 & 2 & 72 & 28 & \multirow[t]{2}{*}{0.001} \\
\hline Non-specialist & 96 & 4 & 84 & 16 & \\
\hline \multicolumn{6}{|l|}{ Previous repair } \\
\hline No & 98 & 2 & 83 & 17 & \multirow[t]{2}{*}{0.002} \\
\hline Yes & 94 & 6 & 64 & 37 & \\
\hline \multicolumn{6}{|l|}{ Mesh type } \\
\hline Synthetic permanent & & & 82 & 18 & \multirow[t]{3}{*}{$<0.001$} \\
\hline Synthetic absorbable & - & - & 25 & 75 & \\
\hline Biologic & & & 0 & 100 & \\
\hline \multicolumn{6}{|l|}{ LOS } \\
\hline 0 & 98 & 2 & 84 & 16 & \multirow[t]{4}{*}{0.001} \\
\hline 1 & 100 & 0 & 74 & 26 & \\
\hline $2-3$ & 90 & 10 & 71 & 29 & \\
\hline$>4$ & 88 & 12 & 37 & 63 & \\
\hline
\end{tabular}

Bold values represent the only variables that were statistically significant (i.e. the result was $<0.05$ )

1.19-4.77), aOR conversion (aOR 3.09, 95\% CI 1.18-8.04), and age (aOR 1.02, 95\% CI 1.01-1.04) were associated with complications at 30 days. Interestingly, increasing age was a predictor of complications with each passing year increasing your risk of 30-day complication by $2 \%(\mathrm{aOR}=1.02,95 \%$ CI 1.001-1.042) (Table 6). 
Table 5 Outcomes characteristics

\begin{tabular}{ll}
\hline Total patients $(n)$ & 312 \\
\hline Length of stay (days); (median, IQ range) & $\mathbf{0}(\mathbf{0}-\mathbf{1})$ \\
0 & 231 \\
1 & 43 \\
$2-3$ & 21 \\
$\geq 4$ & 16 \\
30-day, n (\%) & \\
Post-operative follow-up & $279(89)$ \\
ED presentations & $6(2)$ \\
Readmissions & $6(2)$ \\
C-D Grade for post-operative events (n) & \\
I & 52 \\
II & 3 \\
III & 3 \\
IV & 2 \\
V & 0 \\
30-day complications, n (\%) & $\mathbf{6 0}(\mathbf{1 9})$ \\
Seroma & $52(17)$ \\
Recurrence & $1(0.2)$ \\
Hematoma & $4(1)$ \\
SSI & $2(1)$ \\
DVT & $1(0.2)$ \\
\hline C-D Claven-Dindo, SSI & \\
\hline & \\
&
\end{tabular}

$C-D$ Clavien-Dindo, SSI surgical-site infection, $D V T$ deep venous thrombus, $E D$ Emergency Department

Table 6 Multivariate analysis

\begin{tabular}{llll}
\hline Variables & $p$ value & Odds ratio & $95 \%$ IC \\
\hline Previous repair & $\mathbf{0 . 0 1 4}$ & 2.383 & $1.191-4.770$ \\
Age & $\mathbf{0 . 0 4 1}$ & 1.021 & $1.001-1.042$ \\
OR conversion & $\mathbf{0 . 0 2 1}$ & 3.085 & $1.184-8.038$ \\
Hernia type & 0.276 & - & - \\
ASA & 0.262 & - & - \\
No. of comorbidities & 0.268 & - & - \\
BMI & 0.288 & - & - \\
Type of surgeon & 0.223 & - & - \\
\hline
\end{tabular}

Bold values represent the only variables that were statistically significant (i.e. the result was $<0.05$ )

\section{Discussion}

\section{Significance}

Robotic hernia repair represents the logical next step in the progression of minimally invasive hernia repair. The adoption of robotic surgical technology will continue to be challenged with arguments of high cost and inferior outcomes to other, more established surgical modalities
[20-23]. Despite this, we are beginning to see literature advocating for the adoption of robotic hernia repair based on more favorable outcomes and specific case use scenarios where the robotics platform can provide unique advantages [7, 11, 24]. Looking to the future, platforms that allow multi-quadrant surgery, increasing dexterity, more specialized instrumentation, and less collisions will lead to the increasing adoption of minimally invasive platforms. Furthermore, increasing competition in the robotic surgery market will inevitably drive downs costs, increase the speed at which innovation occurs, and provide more adaptable platforms with less steep learning curves. It is the responsibility of the surgical community to ensure that the adoption of RAWR is safe for our patients, cost effective, and maintains a high standard of care.

\section{Limitations}

This is a retrospective observational study focused on the adoption, intraoperative performance, and short-term outcomes of RAWR at our academic institution. Long-term outcomes data were simply not feasible as more than half of our data represent patients that underwent surgery within the last 18 months and our institution routinely does not follow patients past their initial post-operative visit. However, a study focusing on long-term follow-up is underway at our institution using a validated patient reported outcomes survey. We also did not include a cost analysis of our center's experience since this information was not available to us for unbiased statistical analysis. Finally, having no comparison group can decrease the strength of conclusions drawn from our data. These inherent weaknesses are often part of early, observational, descriptive, outcomes studies.

\section{Adoption and growth}

The proportion of hernia cases approached with the robotics platform steadily increased over the 44-month long study period. Our experience highlighted a slow and steady adoption of RAWR at our institution, paralleling the national trend in nearly the same distribution. Our initial period was marked by a single surgeon performing non-recurrent inguinal hernia repairs and progressed to the creation of an AW program that encouraged an increase in the number of surgeons and difficulty of hernia cases. This growth was facilitated by a multidisciplinary approach to hernia disease alongside colleagues from plastic surgery, bariatric surgery, and radiology. As such, the number of surgeons performing robotic hernia surgery increased sixfold during study period. The difficulty of cases also increased, with the last three quarters of the study period harboring the highest number of complex or combined hernia repair cases. 
In our cohort, we did not find an increase the proportion of complications or intraoperative conversions over time, even as our volume increased. Interestingly, surgery by an expert hernia specialist seemed to confer the patient with an increased risk of 30-day complications on univariate analysis. However, this bias disappeared when accounting for all confounding variables in the regression model and the fact that our expert performed one-third of all of our cases. Thus, even though a hernia specialist takes on a higher proportion of recurrent and otherwise complex hernia cases, their safety and outcomes profile remain comparable to other surgeons.

Interestingly, the proportion of ventral hernia repairs in our cohort was $44 \%$ while that of inguinal hernias was $56 \%$. This proportion is somewhat higher than other published reports where the distribution of ventral to inguinal repairs in a given cohort is closer to $25 \%$ and $75 \%$, respectively. Furthermore, our ventral hernia cohort had a $40 \%$ incidence of extraperitoneal mesh placement, which is higher than seen with traditional laparoscopic cohorts. This may highlight an adoption of the platform with an increased use in more complicated ventral hernia repairs that would otherwise not be attempted laparoscopically.

\section{Intraoperative safety and conversions}

Operative times followed an expected pattern and were noted to be higher with recurrent hernias and complex abdominal wall repairs, like component separation techniques. As expected, the complication rate saw gradual increase that directly paralleled the surgical volume and case complexity but remained comparable to other studies and proportional to the increase in volume. There were two reportable intraoperative events, one of which required a change in the initial operative plan. This was a patient with a history of cirrhosis and controlled portal hypertension who suffered a Veress needle injury to the left lobe of his enlarged liver. This injury required an emergent upper midline laparotomy and repair of hepatic laceration with subsequent admission to the ICU for observation. This complication is not unique to robotic hernia surgery and would have likely been seen with any other minimally invasive approach. Nonetheless, this case highlights the importance of patient selection for minimally invasive approaches to hernia repair. Given the higher risk of complications on abdominal entry carried by patients with cirrhosis, perhaps an open repair would have been a better option for this patient.

The second patient suffered a superficial thermal injury to the sigmoid colon upon reduction of an infraumbilical hernia that was reinforced with sutures and the patient went on to have an uneventful recovery. Given the nature of hernia surgery, small bowel serosal tears were reported in $3 \%$ of cases. No cases reported none further action besides serosal reinforcement, a step facilitated by suturing capabilities of the robotic platform. There were no reported enterotomies.

Three inguinal hernias were converted to an open or hybrid approach; two were recurrent inguinal hernias with previous repairs. In these two cases, an open approach was used to help with reduction of a segment of densely adhered colon to the cord structures and to explant a mesh plug. Both cases were finished robotically with preperitoneal mesh placement as planned. The third was the aborted inguinal hernia secondary to Veress needle injury. The remaining six cases were ventral hernias, four were converted to laparoscopy and two were converted to open. Two had a BMI $>35 \mathrm{~kg} / \mathrm{m}^{2}$ and all but one were females with either umbilical or incisional hernias with multiple fascial defects.

\section{Outcomes}

Robotic hernia repair remains a safe surgical modality with comparable short-term outcomes, even in the early adoption phase. There was a single early recurrence of an umbilical hernia. This patient had a hernia defect of $3 \mathrm{~cm} \times 3 \mathrm{~cm}$, which was repaired with primary closure and reinforced with a $12 \mathrm{~cm}$ Symbotex ${ }^{\circledR}$ mesh that was fixated to the anterior abdominal wall with a running self-locking suture. This patient presented to the emergency department with urinary retention and severe pain at the umbilicus with physical exam findings concerning for recurrence. Confirmatory imaging showed recurrence of hernia with strangulated fat. He was treated with a Foley catheter and analgesic medications and was discharged home and lost to follow-up. This recurrence was likely related to a technical failure of the self-locking suture that was used to fix the mesh to the anterior abdominal wall, a technique that is now far less common at our institution in favor of extraperitoneal mesh placement.

Our rate of intraoperative events $(0.6 \%)$, early recurrence $(0.3 \%)$, and wound related events such as seroma $(17 \%)$, hematoma $(1.2 \%)$, and SSI $(0.6 \%)$ are on par with reported literature for both ventral and inguinal hernias [25]. In our cohort, an infected hematoma required drainage by interventional radiology and a course of oral antibiotics. A second hematoma was drained in the outpatient clinic with no further morbidity. All seromas were treated with supportive treatment and observation.

Overall, we had a low number of OR conversion (3\%) [26], which seemed to be driven more by the complexity of the hernia and less by patient related factors, like as BMI, sex, or ASA. Interestingly, the rate of conversion remained similar even at higher BMI, a trend that is not always seen with laparoscopic modalities and may highlight an advantage of the robotic platform [27]. Nonetheless, our study highlighted that patients with previous hernia repairs and those who had OR conversions were at a statistically higher risk for 30-day complications. 
We also found that increasing age was a risk factor for early complications. In fact, each passing year was related to an increase in 30 -day complication rate by $2.1 \%$. This is probably related to a higher number of comorbid conditions, a higher frequency of recurrence, and larger and more chronic hernias. This supports the notion that delaying surgical care for hernia may increase your overall risk of complications, especially in symptomatic hernias [28].

Safety is a difficult metric to quantify and many factors are considered before a surgical procedure or surgical modality is deemed safe. This is especially true of novel surgical techniques where a direct comparison is not always be possible. Our cohort had a single recurrence, a single major intraoperative event, and a low number of post-operative complications when compared to both the standard of care open techniques and minimally invasive laparoscopic approaches. This remained true for surgeons early in their learning phase, expert surgeons taking on more complex cases, and for patients with a high number of comorbidities, higher BMI's, and those with previous repairs. The safety profile of RAWR is acceptable, especially when you consider the advantages the robotic platform can provide.

\section{Conclusions}

The adoption of robotic-assisted hernia repair is increasing, both among newly trained surgeons and established surgeons. Our initial experience of 312 patients demonstrates favorable results with comparable short-term outcomes for a wide variety of hernia repairs using the robotics platform, even in the initial stages of adoption. Further studies focusing on long-term outcomes, costs, and patient satisfaction are needed.

Author contributions XP: manuscript writing and data collection. DLL: statistical analysis and manuscript writing and editing. CCM: data collection and manuscript writing. GRV: data collection, manuscript editing, tables, and diagrams. VRS: data collection and manuscript writing. AGC: data collection and manuscript writing. PF: statistics and manuscript writing and editing. FM: conceptualization of project, manuscript editing, data analysis, and final approval of submission.

\section{Declarations}

Conflict of interest Author Xavier Pereira, Diego Lima Laurentino, Patricia Friedmann, Cosman Camilo Mandujano, Gustavo RomeroVelez, Vicente Ramos-Santillan, and Ana Garcia-Cabrera declare that they have no conflict of interest". FM received consulting fees from BD, Medtronic \& Intuitive, which are outside the submitted work.

\section{References}

1. Sheetz KH, Claflin J, Dimick JB (2020) Trends in the adoption of robotic surgery for common surgical procedures. JAMA Netw Open 3(1):e1918911. https://doi.org/10.1001/jamanetworkopen. 2019.18911

2. Bittner R, Bain K, Bansal VK et al (2019) Update of Guidelines for laparoscopic treatment of ventral and incisional abdominal wall hernias (International Endohernia Society (IEHS)): Part B. Surg Endosc 33(11):3511-3549. https://doi.org/10.1007/ s00464-019-06908-6

3. Giulianotti PC, Coratti A, Angelini M et al (2003) Robotics in general surgery: personal experience in a large community hospital. Arch Surg Chic Ill 1960. 138(7):777-784. https://doi. org/10.1001/archsurg.138.7.777

4. Aggarwal R, Hance J, Darzi A (2004) Robotics and surgery: a long-term relationship? Int J Surg Lond Engl 2(2):106-109. https://doi.org/10.1016/S1743-9191(06)60055-1

5. Pahwa HS, Kumar A, Agarwal P, Agarwal AA (2015) Current trends in laparoscopic groin hernia repair: a review. World J Clin Cases. https://doi.org/10.12998/wjcc.v3.i9.789

6. Donkor C, Gonzalez A, Gallas M, Helbig M, Weinstein C, Rodriguez J (2017) Current perspectives in robotic hernia repair. Robot Surg Res Rev 4:57-67. https://doi.org/10.2147/RSRR. S101809

7. Podolsky D, Novitsky Y (2020) Robotic Inguinal Hernia Repair. Surg Clin N Am 100(2):409-415. https://doi.org/10.1016/j.suc. 2019.12.010

8. Abdelmoaty WF, Dunst CM, Neighorn C, Swanstrom LL, Hammill CW (2019) Robotic-assisted versus laparoscopic unilateral inguinal hernia repair: a comprehensive cost analysis. Surg Endosc 33(10):3436-3443. https://doi.org/10.1007/s00464-018-06606-9

9. Gonzalez A, Escobar E, Romero R et al (2017) Robotic-assisted ventral hernia repair: a multicenter evaluation of clinical outcomes. Surg Endosc 31(3):1342-1349. https://doi.org/10.1007/ s00464-016-5118-0

10. Kudsi OY, Gokcal F, Chang K (2020) Robotic intraperitoneal onlay versus totally extraperitoneal (TEP) retromuscular mesh ventral hernia repair: a propensity score matching analysis of short-term outcomes. Am J Surg. https://doi.org/10.1016/j. amjsurg.2020.01.003 (Published online January 8, 2020)

11. Goettman MA, Riccardi ML, Vang L, Dughayli MS, Faraj CH (2020) Robotic assistance in ventral hernia repair may decrease the incidence of hernia recurrence. J Minimal Access Surg. https://doi.org/10.4103/jmas.JMAS_92_19 (Published online January 3, 2020)

12. Sharbaugh ME, Patel PB, Zaman JA et al (2019) Robotic ventral hernia repair: a safe and durable approach. Hernia J Hernias Abdom Wall Surg. https://doi.org/10.1007/s10029-019-02074-9 (Published online November 27, 2019)

13. County Health Rankings. University of Wisconsin Population Health Institute. 2020 County health rankings report: New York. Accessed April 13, University of Wisconsin Population Health Institute. 2020 County health rankings report: New York. https://a816-health.nyc.gov/hdi/profiles/. Accessed 5 Aug 2020

14. Bronx County, New York. County Health Rankings \& Roadmaps. https://www.countyhealthrankings.org/app/new-york/ 2020/rankings/bronx/county/outcomes/overall/snapshot. Accessed 5 Aug 2020

15. Charlson ME, Pompei P, Ales KL, MacKenzie CR (1987) A new method of classifying prognostic comorbidity in longitudinal studies: development and validation. J Chronic Dis 40(5):373383. https://doi.org/10.1016/0021-9681(87)90171-8

16. Köckerling F, Berger D, Jost JO (2014) What is a certified hernia center? The example of the German Hernia Society and 
German Society of General and Visceral Surgery. Front Surg 1:26. https://doi.org/10.3389/fsurg.2014.00026

17. Köckerling F, Sheen AJ, Berrevoet F et al (2019) Accreditation and certification requirements for hernia centers and surgeons: the ACCESS project. Hernia 23(2):185-203. https://doi.org/10.1007/ s10029-018-1873-2

18. Dindo D, Demartines N, Clavien P-A (2004) Classification of surgical complications: a new proposal with evaluation in a cohort of 6336 patients and results of a survey. Ann Surg 240(2):205-213. https://doi.org/10.1097/01.sla.0000133083.54934.ae

19. Ebeling PA, Beale KG, Van Sickle KR et al (2020) Resident training experience with robotic assisted transabdominal preperitoneal inguinal hernia repair. Am J Surg 219(2):278-282. https://doi.org/ 10.1016/j.amjsurg.2019.11.014

20. Jeong IG, Khandwala YS, Kim JH et al (2017) Association of robotic-assisted vs laparoscopic radical nephrectomy with perioperative outcomes and health care costs, 2003 to 2015. JAMA 318(16):1561-1568. https://doi.org/10.1001/jama.2017.14586

21. Wright JD, Ananth CV, Lewin SN et al (2013) Robotically assisted vs laparoscopic hysterectomy among women with benign gynecologic disease. JAMA 309(7):689-698. https://doi.org/10.1001/ jama.2013.186

22. Prabhu AS, Carbonell A, Hope W et al (2020) Robotic inguinal vs transabdominal laparoscopic inguinal hernia repair: the RIVAL randomized clinical trial. JAMA Surg. https://doi.org/10.1001/ jamasurg.2020.0034 (Published online March 18, 2020)

23. Ramirez PT, Frumovitz M, Pareja R et al (2018) Minimally invasive versus abdominal radical hysterectomy for cervical cancer. N Engl J Med 379(20):1895-1904. https://doi.org/10.1056/NEJMo a1806395
24. Fuenmayor P, Lujan HJ, Plasencia G, Karmaker A, Mata W, Vecin N (2020) Robotic-assisted ventral and incisional hernia repair with hernia defect closure and intraperitoneal onlay mesh (IPOM) experience. J Robot Surg. https://doi.org/10.1007/s11701019-01040-y (Published online January 2, 2020)

25. Olsen MA, Nickel KB, Wallace AE, Mines D, Fraser VJ, Warren DK (2015) Stratification of surgical site infection by operative factors and comparison of infection rates after hernia repair. Infect Control Hosp Epidemiol 36(3):329-335. https://doi.org/10.1017/ ice.2014.44

26. Kudsi OY, Paluvoi N, Bhurtel P, McCabe Z, El-Jabri R (2015) Robotic repair of ventral hernias: preliminary findings of a case series of 106 consecutive cases. Am J Robot Surg 2(1):22-26. https://doi.org/10.1166/ajrs.2015.1020

27. Zanghì A, Di Vita M, Borzì $\mathrm{A}$ et al (2018) Laparoscopic repair of incisional hernia. Risk factors for the conversion. Ann Ital Chir. $89: 242-246$

28. Gong W, Li J (2018) Operation versus watchful waiting in asymptomatic or minimally symptomatic inguinal hernias: the metaanalysis results of randomized controlled trials. Int J Surg Lond Engl 52:120-125. https://doi.org/10.1016/j.ijsu.2018.02.030

Publisher's Note Springer Nature remains neutral with regard to jurisdictional claims in published maps and institutional affiliations. 